\title{
PROPUESTA DIDÁCTICA DE ATLETISMO BASADA EN EL MODELO DE PROYECTOS Y LA AUTORREGULACIÓN
}

Proposal of learning athletics based on Project based learning (PBL) and self-regulation methodologies

Atletismo de ensino proposta baseada projeto de modelo e auto-regulação

\section{Carlos Heras Bernardino}

\section{Ángel Pérez Pueyo}

\section{Israel Herrán Álvarez}

(1) IES Prado de Santo Domingo. carlos.herasbernardino@educa.madrid.org

(2) Universidad de León. angel.perez.pueyo@unileon.es

(3). IES Doctor Sancho de Matienzo. iherran@educa.jcyl.es

\begin{abstract}
Resumen
A continuación se presenta una propuesta didáctica de Atletismo para la materia de educación física en $1^{\circ}$ ESO, basada en la metodología de proyectos y la autorregulación, donde la clave reside en conseguir que todos los alumnos tengan experiencias positivas y de éxito. Teniendo como finalidad/producto final el diseño y participación en una competición de atletismo, los alumnos realizan un trabajo colaborativo en el cual autorregulan y gestionan su propio aprendizaje. El trabajo se inicia con el diseño de un planificador grupal, partiendo de plantillas con secuencias de tareas para cada una de las pruebas aportadas por el profesor. Todo ello, asociado a procesos de evaluación formativa y compartida integrados en el proceso de enseñanza-aprendizaje.

Palabras clave: Autorregulación; Evaluación formativa; Atletismo; Educación física
\end{abstract}

\begin{abstract}
Our proposal of learning Athletics is based on both Project based learning (PBL) and self-regulation methodologies, while the focus is placed on providing positive and successful experiences to all our students. The final goal is to design an athletic competition and also participate on it. The students develop a collaborative task in which the self-regulate and manage their own learning. This proposal starts with the Propuesta didáctica de atletismo basada en el modelo de proyectos y la autorregulación
\end{abstract}


design of a group planner, following different templates provided by the teacher with sequences of tasks to follow for each of the tests. All of it, related to shared and formative assessment processes fully integrated in the teaching-learning process.

Keywords: Self-regulation; Formative assessment; Athletics; Physical education

\section{Resumo}

A experiência recolhida Abaixo está uma didáticos Atletismo proposta baseada na metodologia de projecto e de auto-regulação, em que a chave é assegurar que todos os alunos têm experiências positivas e bem sucedidas. Tomando Design Alvo / produto final e participação em competição de atletismo, os alunos realizam o trabalho colaborativo em que a auto-regular e gerir a sua própria aprendizagem. O trabalho começa com o desenho de um agendador de grupo, com base em modelos com seqüências de tarefas para cada um dos elementos de prova apresentados pelo professor. Tudo isso, combinado com treinamento e compartilhados processos integrados na avaliação do processo de ensino-aprendizagem.

Palavras-chave: Autorregulacón; Avaliação formativa; Atletismo, Educação física

\section{Introducción}

La siguiente experiencia recoge una propuesta didáctica para $1^{\circ} \mathrm{ESO}$ relacionada con contenidos de Atletismo en la materia de educación física. Tomando como base el Estilo Actitudinal (Pérez-Pueyo, 2005), la propuesta gira en torno a tres ejes fundamentales:

- La metodología basada en proyectos, donde el componente grupal del aprendizaje permite a los alumnos un trabajo de su capacidad para dialogar, discutir y ponerse de acuerdo, así como el respeto a normas.

- Las estrategias vinculadas a la autorregulación (Zimmerman \& Schunk, 1990, Tomlinson, 2003, Torrano-Montalvo, 2004; ...), que generan un papel activo del alumno en la toma de decisiones y gestión de su aprendizaje.

- Y por último la evaluación formativa y compartida: mediante procesos de autoevaluación a través de un diario de seguimiento intragrupal, y coevaluación vinculados a la ejecución técnica de cada prueba. 


\section{Contextualización}

La propuesta ha sido diseñada e implementada en tres grupos de $1^{\circ} \mathrm{ESO}$ de un centro situado en una ciudad localizada al sur de la comunidad de Madrid, caracterizado por presentar un proyecto propio denominado "IES de especialización deportiva”. Cuenta con cuatro horas semanales en el área de Educación Física y una optativa de Deportes a lo largo de toda la ESO.

\section{Diseño y desarrollo}

Siguiendo las bases de la metodología basada en proyectos, la propuesta se organiza en cuatro fases fundamentales (Figura 1).

\begin{tabular}{|c|c|c|}
\hline Sesión 1 & \multicolumn{2}{|c|}{$\begin{array}{l}\text { Fase 1: presentación del producto final y } \\
\text { análisis de necesidades de aprendizaje }\end{array}$} \\
\hline Sesión 2 & \multicolumn{2}{|c|}{ Fase 2: Diseño grupal de los planificadores } \\
\hline Sesiones 3-7 & \multicolumn{2}{|c|}{$\begin{array}{l}\text { Fase 3: puesta en práctica grupal del } \\
\text { planificador }\end{array}$} \\
\hline \multirow{3}{*}{ Sesiones 8-11 } & \multirow{3}{*}{$\begin{array}{l}\text { Fase 4: } \\
\text { Elaboración } \\
\text { del } \\
\text { producto } \\
\text { final }\end{array}$} & $\begin{array}{l}\text { Sesiones 8-9: práctica global de } \\
\text { las pruebas: mediciones y } \\
\text { coevaluación técnica }\end{array}$ \\
\hline & & $\begin{array}{l}\text { Sesión 10: organización de la } \\
\text { competición (grupos de } \\
\text { expertos por prueba) }\end{array}$ \\
\hline & & $\begin{array}{l}\text { Sesión 11: desarrollo de la } \\
\text { competición interna }\end{array}$ \\
\hline
\end{tabular}

Figura 1.

Temporalización de la unidad didáctica

La primera de las fases comienza presentando el producto final de la unidad: diseñar y organizar de manera colaborativa una competición de atletismo (interna en el grupoclase) que incluyan las siguientes pruebas: lanzamiento de peso, salto de longitud, 50 metros, 4x50m y 4x200m.

Una vez definido el producto final, corresponde analizar con los alumnos las necesidades de aprendizaje. Para ello, mediante el visionado de videos preparados previamente por el profesor, se da a conocer las pruebas que van a desarrollar, se analizan aspectos técnicos básicos, y se valoran sus experiencias previas.

Ya en la segunda fase, siguiendo estrategias vinculadas a la autorregulación, cada grupo (5-6 alumnos) elabora el plan de trabajo, que recogen en un planificador grupal (figura 2). Para ayudarles en este proceso, el profesor prepara unas fichas de cada 
prueba en la que se recogen: resumen de los aspectos técnicos más importantes, imágenes del gesto técnico y una propuesta de actividades vinculadas al aprendizaje de la misma (figura 3). En el planificador dejan definido qué secuencia de actividades, de entre las presentadas en las fichas, van a desarrollar en cada sesión de la fase 3 (no más de dos pruebas por día).

\begin{tabular}{|c|c|c|c|c|c|}
\hline & \multicolumn{2}{|c|}{ PRUEBA A PREPARAR } & \multicolumn{2}{|c|}{ PRUEBA A PREPARAR } & \multirow[b]{2}{*}{$\begin{array}{c}\text { Comentarios/valoración } \\
\text { del cumplimiento del } \\
\text { planificador }\end{array}$} \\
\hline & $\begin{array}{l}\text { Secuencia } \\
\text { de tareas } \\
\text { principal }\end{array}$ & $\begin{array}{l}\text { Secuencia de } \\
\text { tareas } \\
\text { principal }\end{array}$ & $\begin{array}{l}\text { Secuencia de } \\
\text { tareas } \\
\text { secundaria }\end{array}$ & $\begin{array}{l}\text { Secuencia de } \\
\text { tareas } \\
\text { secundaria }\end{array}$ & \\
\hline \multicolumn{6}{|l|}{ Sesión 1} \\
\hline \multicolumn{6}{|l|}{ Sesión 2} \\
\hline \multicolumn{6}{|l|}{ Sesión 3} \\
\hline \multicolumn{6}{|l|}{ Sesión 4} \\
\hline Sesión 5 & & & & & \\
\hline
\end{tabular}

Figura 2.

Planificador grupal

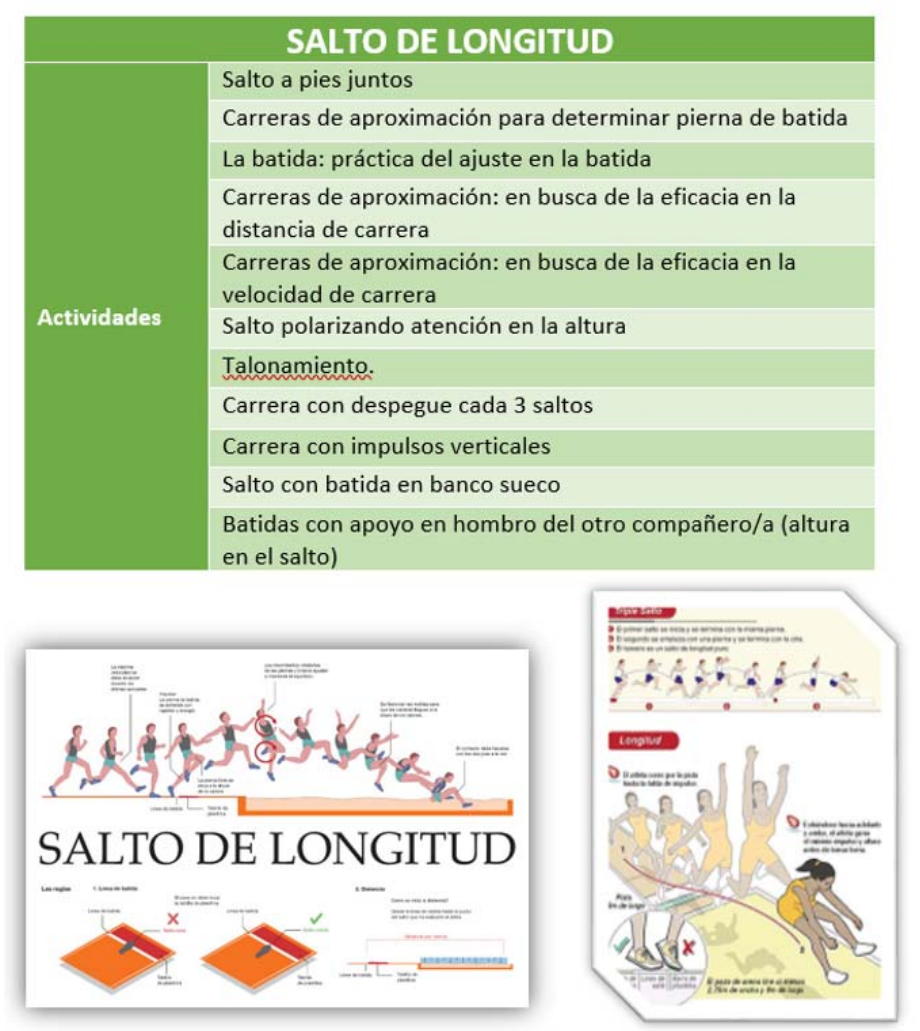

Figura 3.

Ejemplo de ficha de trabajo: salto de longitud

Propuesta didáctica de atletismo basada en el modelo de proyectos y la autorregulación 
Durante la fase 3 (puesta en práctica, sesiones 3-7) cada grupo trabaja de manera autónoma en base a lo establecido en su planificador, gestionando el material, los espacios, los tiempos, las tareas y el propio grupo. Al final de cada sesión realizan una autoevaluación intragrupal del trabajo diario.

Por último, la fase de elaboración del producto final (sesiones 8-11) se desarrolla en tres momentos de aprendizaje fundamentales:

- El primero consiste en definir la participación de los alumnos en las pruebas. Para ello, durante las sesiones 8 y 9 cada uno de los grupos debe practicar globalmente todas las pruebas registrando en su planificador los datos y mediciones obtenidas por cada integrante del grupo. Esta información les permitirá consensuar de manera crítica quién realiza cada una de las pruebas (siguiendo las normas recogidas en la Tabla 1). Estas sesiones se aprovechan a su vez para realizar un proceso de coevaluación intragrupal sobre aspectos técnicos en cada una de las pruebas.

Tabla 1.

Normas para la participación en la competición

\section{Normas para la participación en la competición}

Participarán 2 alumnos de cada grupo en cada prueba individual (salto de longitud, lanzamiento de peso y $50 \mathrm{ML}$ )

Todos los miembros del grupo participarán al menos en una de las pruebas de relevos

Se puntuará cada prueba sumando los tiempos/distancias de los dos miembros del grupo

- El segundo consiste en la propia organización de la competición, gestionada mediante grupos de expertos de cada prueba (alumnos que van a participan en dicha prueba), que se encargan de determinar las normas (número de intentos, orden de participación, etc.) y diseñar la plantilla de registro de datos.

- El tercero implica el desarrollo y participación en la propia competición, momento en el que los alumnos disfrutarán de lo que ellos mismos han generado como resultado de su aprendizaje.

\section{Evaluación}

La propuesta de evaluación se caracteriza por su carácter formativo y compartido y por estar integrada en el proceso de enseñanza aprendizaje. 
Destaca el uso del diario de seguimiento intragrupal (durante la fase de puesta en práctica), en el cual cada grupo valora de manera conjunta el trabajo realizado por cada uno de sus miembros (Figura 4).

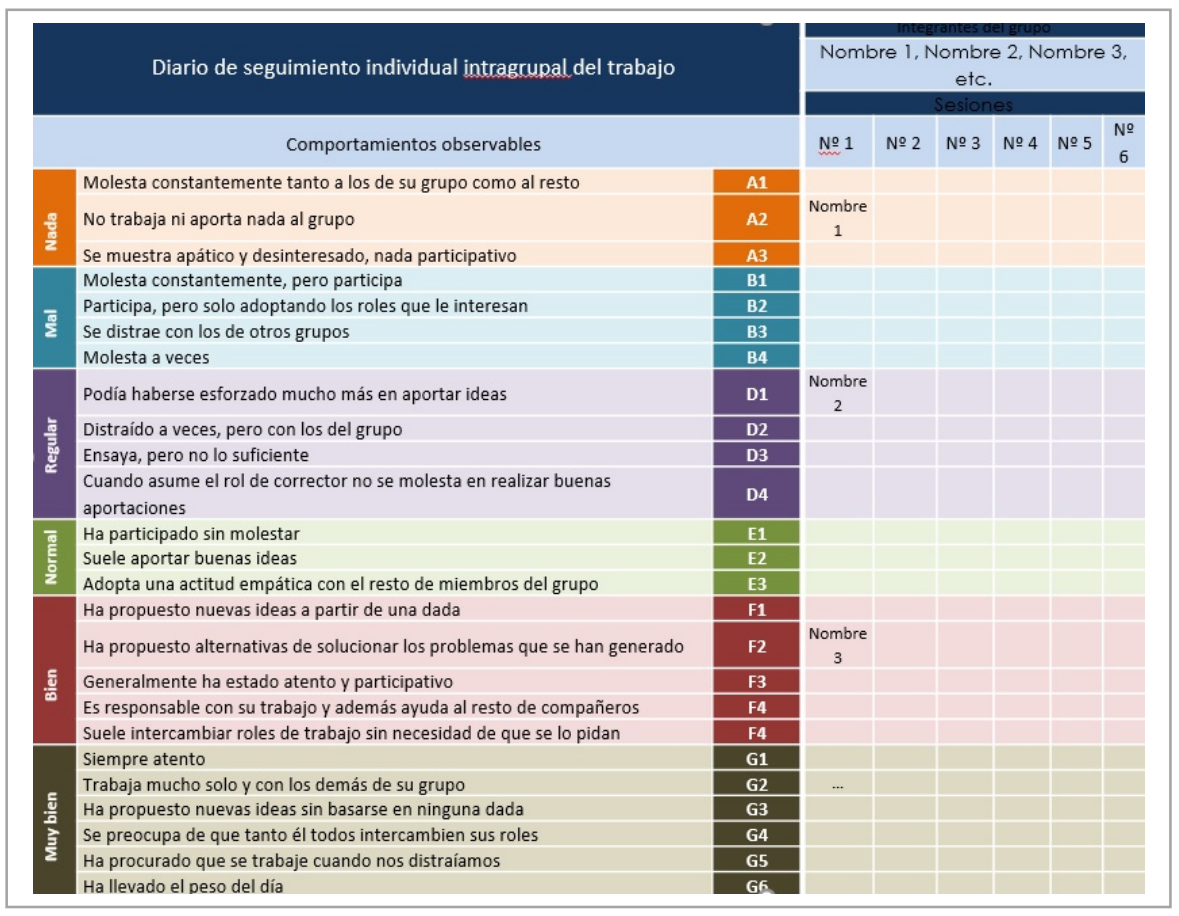

Figura 4.

Diario de Seguimiento Intragrupal (modelo de Grupo Actitudes)

El planificador se convierte en un instrumento muy valioso para la evaluación formativa. En él los grupos van recopilando una valoración general del desarrollo de la en cada sesión y del cumplimiento de lo establecido en el planificador (autorregulación de su aprendizaje), que es revisado por el profesor durante el desarrollo de cada una de las sesiones.

Por otro lado, durante las sesiones 8 y 9 los alumnos desarrollan un proceso de coevaluación intragrupal sobre aspectos técnicos. Para ello disponen de rúbricas aportadas por el profesor (figura 5). Finalmente para la valoración del trabajo diario se emplea un registro anecdótico (Pérez-Pueyo, 2013) (Figura 6).

\section{Conclusiones}

La implicación del alumnado en la toma decisiones, así como la gestión colectiva en el cumplimiento de lo planificado (autorregulación), han generado una mejora del clima de clase. Además, las estrategias de autorregulación han permitido polarizar la atención del alumno en la tarea, reduciendo considerablemente las pérdidas de tiempo y los Propuesta didáctica de atletismo basada en el modelo de proyectos y la autorregulación 
problemas generados en las explicaciones colectivas. En definitiva, la propuesta ha contribuido a que los alumnos aprendan a ser autónomos, generando experiencias positivas y de éxito en todos los alumnos.

\begin{tabular}{|c|c|}
\hline \multicolumn{2}{|l|}{ ALUMANO/A QUE LANZA: } \\
\hline ALUMANOS QUE EVALULAN: & 10 EsO: \\
\hline \multicolumn{2}{|l|}{ 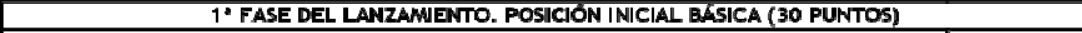 } \\
\hline $\begin{array}{l}\text { El agarre de la bola se realiza correctamente colocando los } 3 \text { dedos centrales sujetando la } \\
\text { mayor parte de la bola y el pulgar y el indice por abato para que no se calga }\end{array}$ & 10 puntos \\
\hline $\begin{array}{l}\text { La colocación de la bola se realiza debajo de la mandibula y no detrás de la cabeza } \\
\text { colocándose de espaldas a la zona donde caerá la bola. }\end{array}$ & 10 puntos \\
\hline 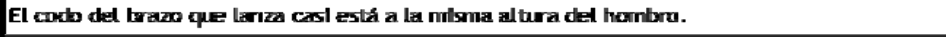 & 5 pantes \\
\hline 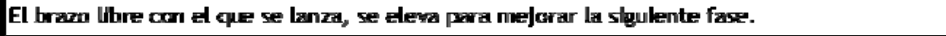 & 5 pantes \\
\hline \multicolumn{2}{|l|}{ 2 FASE DEL LANZAMIENTO. DESLIZAMAIEMTO (45 PUNTOS) } \\
\hline $\begin{array}{l}\text { El movimiento de agrupamiento es rápido y flexiona la plerna del mismo lado del brazo que } \\
\text { lanza. }\end{array}$ & 5 puntos \\
\hline $\begin{array}{l}\text { La plema del lado del brazo libre se levanta del sulelo y prepara para dar un paso amplio } \\
\text { hacla atrás. }\end{array}$ & 5 puntos \\
\hline $\begin{array}{l}\text { El salto que se reallza hacka atrás es lo más amplio posible y el primer ple en tocar el suelo } \\
\text { es el mlsmo del lado de la mano que lanza. }\end{array}$ & 10 puntos \\
\hline $\begin{array}{l}\text { Después del primer contacto con el suelo, la plema que estaba en el aire da un gran paso } \\
\text { hacla atrás para arcercarse lo más posible al limite del circulo de lanzamiento. }\end{array}$ & 10 puntos \\
\hline La bola no se ha separado en ntrgün mornento del cuello. & 15 puncoss \\
\hline \multicolumn{2}{|l|}{ 332 FASE DEL LANEAMENTO (25 PUNTOS) } \\
\hline $\begin{array}{l}\text { Una yez han contactado los } 2 \text { pdes en el suelo se produce un giro hacla el lado del brazo que } \\
\text { lanza para soltar la bola con el mayor impulso. }\end{array}$ & 5 puntos \\
\hline $\begin{array}{l}\text { El brazo que lanza suelta la bola haclendo una extensión completa y sin separar la bola de } \\
\text { la mandflbula hasta el último momento. }\end{array}$ & 15 puntos \\
\hline $\begin{array}{l}\text { La plema del mismo lado del brazo que lanza, se adelanta para frenar el movmiento y no } \\
\text { salirse del circulo. }\end{array}$ & 5 puntos \\
\hline PUNTIUACIÓN TOTAL & \\
\hline
\end{tabular}

Figura 5.

Rúbrica de coevaluación de la técnica de lanzamiento

\begin{tabular}{|c|c|c|c|c|c|}
\hline ALumNos'AS & $\begin{array}{l}\text { PAR TIFAPCÓN } \\
\text { ACTMAEN EN LA } \\
\text { SESSION }\end{array}$ & 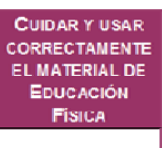 & 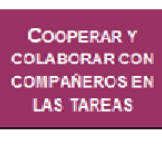 & 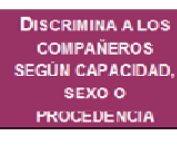 & 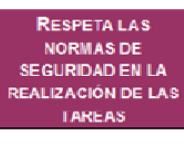 \\
\hline 2. & & & & & \\
\hline 3. & & & & & \\
\hline$\frac{4 .}{5}$ & & & & & \\
\hline$\frac{5 .}{6 .}$ & & & & & \\
\hline & & & & & \\
\hline 8. & & & & & \\
\hline$\frac{9 .}{10}$ & & & & & \\
\hline$\frac{10 .}{11 .}$ & & & & & \\
\hline 12 & & & & & \\
\hline 13. & & & & & \\
\hline$\frac{14 .}{15}$ & & & & & \\
\hline 16. & & & & & \\
\hline & & & & & \\
\hline$\frac{18 .}{10 .}$ & & & & & \\
\hline 20. & & & & & \\
\hline 21. & & & & & \\
\hline & & & & & \\
\hline 24. & & & & & \\
\hline & & & & & \\
\hline
\end{tabular}

Figura 6.

Registro anecdótico

Propuesta didáctica de atletismo basada en el modelo de proyectos y la autorregulación 


\section{Referencias}

Casado, O. M., \& Pérez-Pueyo, A. (2016). Diario de Seguimiento Intragrupal. Recuperado de https://drive.google.com/file/d/0B7xEdPlO4dj8TEZWSmZVdlhFZ2c/view

Pérez-Pueyo, Á. (coord.). (2013). Programar y evaluar competencias en 15 pasos. Barcelona: Graó.

Pérez-Pueyo, A. (2010). El Estilo Actitudinal. Una propuesta metodológica para desarrollar unidades didácticas en educación física. Madrid: CEP

Tomlinson, C. A. (2003). El aula diversificada. Barcelona: Octaedro.

Torrano-Montalvo, F. (2004). El aprendizaje autorregulado: presente y futuro de la investigación. Pamplona: Universidad de Navarra, Departamento de Educación.

Zimmerman, B.J., \& Schunk, D.H. (1990). Self-regulated learning and academic achievement. Educational Psychologist, 25(1), 3-17. 